\title{
FINITE NONABELIAN $p$-GROUPS OF EXPONENT $>p$ WITH A SMALL NUMBER OF MAXIMAL ABELIAN SUBGROUPS OF EXPONENT $>p$
}

\author{
ZVONIMIR JANKO \\ University of Heidelberg, Germany
}

\begin{abstract}
Y. Berkovich has proposed to classify nonabelian finite $p$ groups $G$ of exponent $>p$ which have exactly $p$ maximal abelian subgroups of exponent $>p$ and this was done here in Theorem 1 for $p=2$ and in Theorem 2 for $p>2$. The next critical case, where $G$ has exactly $p+1$ maximal abelian subgroups of exponent $>p$ was done only for the case $p=2$ in Theorem 3 .
\end{abstract}

Let $G$ be a nonabelian finite $p$-group of exponent $>p$. If $S$ is a minimal nonabelian subgroup in $G$, then $S$ has exactly $p+1$ maximal subgroups $S_{1}, S_{2}, \ldots, S_{p+1}$ and they are abelian and they lie in $p+1$ pairwise distinct maximal abelian subgroups in $G$. If at least two of $S_{i}^{\prime} s$ are elementary abelian, then $S$ is generated by its elements of order $p$ and then (by Lemma 65.1 in [2]) $S \cong \mathrm{D}_{8}$ or $S \cong \mathrm{S}\left(p^{3}\right)$ (the nonabelian group of order $p^{3}$ and exponent $p>2$ ). If all minimal nonabelian subgroups of $G$ are generated by its elements of order $p$, then by Theorem 10.33 in [1] (for $p=2$ ) and Proposition 7 in [3] (for $p>2), G$ has only one maximal abelian subgroup $A$ of exponent $>p$, where $A$ is of index $p$ in $G$ and $A=\mathrm{H}_{p}(G)$ (Hughes subgroup). However, if a minimal nonabelian subgroup of $G$ has at most one elementary abelian maximal subgroup, then $G$ has at least $p$ maximal abelian subgroups of exponent $>p$.

From the above follows that a nonabelian $p$-group $G$ of exponent $>p$ has either exactly one maximal abelian subgroup of exponent $>p$ or $G$ has at least $p$ of them. Therefore Y. Berkovich has proposed to classify nonabelian finite $p$-groups of exponent $>p$ which have exactly $p$ maximal abelian subgroups of exsponent $>p$ and this was done here in Theorem 1 for $p=2$ and in Theorem

2010 Mathematics Subject Classification. 20D15.

Key words and phrases. Finite p-groups, minimal nonabelian subgroups, maximal abelian subgroups, quasidihedral 2-groups, Hughes subgroup. 
2 for $p>2$. By the above, such a group $G$ possesses a minimal nonabelian subgroup $S$ which is not isomorphic to $\mathrm{D}_{8}$ or $\mathrm{S}\left(p^{3}\right)$. Also, such an $S$ has exactly one maximal subgroup $X$ which is elementary abelian so that $\Phi(S)=\mathrm{Z}(S)$ is elementary abelian and $|S: \Phi(S)|=p^{2}$. Let $a \in S-X$ and $b \in X-\Phi(S)$ so that $\mathrm{o}(a) \leq p^{2}, \mathrm{o}(b)=p$ and $S=\langle a, b\rangle$, where $\Phi(S)=\left\langle a^{p},[a, b]\right\rangle$. If $|\Phi(S)|=p$, then $|S|=p^{3}$ and $S \cong \mathrm{M}_{p^{3}}$ (the nonabelian group of order $p^{3}$ and exponent $p^{2}$, where $p>2$ ). If $|\Phi(S)|=p^{2}$, then $S \cong \mathrm{M}_{p}(2,1,1)$, where

$$
\mathrm{M}_{p}(2,1,1)=\left\langle a, b \mid a^{p^{2}}=b^{p}=1,[a, b]=c, c^{p}=[c, a]=[c, b]=1\right\rangle .
$$

Suppose that $G$ possesses a non-normal maximal abelian subgroup $H$ of exponent $>p$. Set $K=\mathrm{N}_{G}(H)$ so that $|G: K|=p, H<K$ and $H^{G} \leq K$. All elements in $G-K$ are of order $p$. If $p=2$, then $K$ is abelian (by a result of Burnside), a contradiction. Hence in this case we must have $p>2$. For any $g \in G-K, H^{g} \leq K$ and so $H$ and $H^{g}$ normalize each other.

Y. Berkovich has proposed to consider also the next critical case, where $G$ has exactly $p+1$ maximal abelian subgroups of exponent $>p$. However, we have been able to classify such $p$-groups only in case $p=2$ in Theorem 3 .

TheOREM 1. Let $G$ be a nonabelian 2-group with exactly 2 maximal abelian subgroups of exponent $>2$. Then $G=M \times V$, where

$$
M \cong \mathrm{M}_{2}(2,1,1)=\left\langle a, b \mid a^{4}=b^{2}=1,[a, b]=c, c^{2}=[c, a]=[c, b]=1\right\rangle
$$

and $\exp (V) \leq 2$.

Proof. Let $G$ be a nonabelian 2-group with exactly 2 maximal abelian subgroups of exponent $>2$. Let $H_{1}$ and $H_{2}$ be the two maximal abelian subgroups of exponent $>2$, where we know that $H_{1}$ and $H_{2}$ are normal in $G$. If $H_{1} H_{2}<G$, then all elements in $G-\left(H_{1} H_{2}\right)$ are involutions and then (by a result of Burnside) $H_{1} H_{2}$ would be abelian, a contradiction. Hence $H_{1} H_{2}=G$ and $H_{1} \cap H_{2}=\mathrm{Z}(G)$ so that $G$ is of class 2 and all elements in $G-\left(H_{1} \cup H_{2}\right)$ are involutions. Indeed, all elements of order $>2$ lie in $H_{1}$ or $H_{2}$ (by our hypothesis). If $g \in G-\left(H_{1} \cup H_{2}\right)$, then a maximal abelian subgroup $H$ containing $\langle g\rangle$ is elementary abelian implying that $\mathrm{Z}(G)$ is elementary abelian. Since $H \unlhd G$, Lemma 57.1 in [2] implies that for any $x \in G-H$ there is $h \in H$ such that $\langle x, h\rangle$ is minimal nonabelian. Since $\langle x, h\rangle \cong \mathrm{D}_{8}$ or $\mathrm{M}_{2}(2,1,1)$, it follows that $\exp (\langle x, h\rangle)=4$ and so o $(x) \leq 4$. We have proved that $\exp (G)=4$. For any $x, y \in G,\left[x^{2}, y\right]=[x, y]^{2}=1$ and so we get $\mho_{1}(G) \leq \mathrm{Z}(G)$.

Suppose that both $H_{1}$ and $H_{2}$ are not maximal subgroups in $G$. Then $\left|H_{i}: \mathrm{Z}(G)\right| \geq 4$ for $i=1,2$ and let $h_{i} \in H_{i}-\mathrm{Z}(G)$ be an element of order $4(i=1,2)$ so that $1 \neq h_{i}^{2} \in \mathrm{Z}(G)$. Let $H_{i}^{*}$ be a maximal subgroup of $H_{i}$ which contains $\mathrm{Z}(G)\left\langle h_{i}\right\rangle, i=1,2$. Then $M_{1}=H_{1} H_{2}^{*}$ and $M_{2}=H_{2} H_{1}^{*}$ are distinct maximal subgroups of $G$ containing $H_{1}$ and $H_{2}$, respectively. Since all elements in $G-\left(H_{1} \cup H_{2}\right)$ are involutions, it follows that all elements in 
$G-\left(M_{1} \cup M_{2}\right)$ are involutions. Let $g \in G-\left(M_{1} \cup M_{2}\right)$ and $m \in M_{1} \cap M_{2}$. Then $g$ and $g m \in G-\left(M_{1} \cup M_{2}\right)$ are involutions and so we get

$$
1=(g m)^{2}=g m g m=g^{2} m^{g} m=m^{g} m \text { and so } m^{g}=m^{-1} .
$$

It follows that $g$ inverts each element in $M_{1} \cap M_{2}$ so that a result of Burnside implies that $M_{1} \cap M_{2}$ is abelian. In particular, $\left\langle h_{1}, h_{2}\right\rangle$ is abelian. Let $Y$ be a maximal abelian subgroup in $G$ containing $\left\langle h_{1}, h_{2}\right\rangle$. By our hypothesis, $Y=H_{1}$ or $Y=H_{2}$, a contradiction. We have proved that we may assume $\left|G: H_{1}\right|=2$ and so $H_{1}$ is a maximal subgroup in $G$.

Let $H_{1}^{*}$ be a maximal subgroup of $H_{1}$ containing $\Omega_{1}\left(H_{1}\right)$. Then $M_{2}=$ $H_{2} H_{1}^{*}$ is a maximal subgroup of $G$ and all elements in $G-\left(H_{1} \cup M_{2}\right)$ are involutions. If $g \in G-\left(H_{1} \cup M_{2}\right)$, then for any $x \in H_{1}^{*}=H_{1} \cap M_{2}, g x \in$ $G-\left(H_{1} \cup M_{2}\right)$ is an involution. This implies $x^{g}=x^{-1}$ and so $g$ inverts each element in $H_{1}^{*}$. In particular, $g$ centralizes $\Omega_{1}\left(H_{1}\right)$. It follows that $\Omega_{1}\left(H_{1}\right) \leq \mathrm{Z}(G)$ and so $\Omega_{1}\left(H_{1}\right)=\mathrm{Z}(G)=H_{1} \cap H_{2}$ and therefore all elements in $H_{1}-\mathrm{Z}(G)$ are of order 4 .

Suppose that $\mathrm{Z}(G)$ is not a maximal subgroup in $H_{1}$. Note that all elements in $G-\left(H_{1} \cup H_{2}\right)$ are involutions and all elements in $H_{2}-H_{1}$ and in $H_{1}-\mathrm{Z}(G)$ are of order 4 . Let $v \in H_{1}-\mathrm{Z}(G)$ so that $v^{2} \in \mathrm{Z}(G)$ and let $H_{1}^{* *}$ be a maximal subgroup of $H_{1}$ containing $\mathrm{Z}(G)\langle v\rangle$ so that $M_{2}^{*}=H_{1}^{* *} H_{2}$ is a maximal subgroup in $G$. If $g \in G-\left(H_{1} \cup M_{2}^{*}\right)$, then $g$ and $g v \in G-\left(H_{1} \cup M_{2}^{*}\right)$ are involutions implying that $v^{g}=v^{-1}$. Then each element in $G-H_{1}$ also inverts $\langle v\rangle$. Hence each element in $G-H_{1}$ inverts each element of order 4 in $H_{1}$ and since it also centralizes $\mathrm{Z}(G)$, it follows that each element in $G-H_{1}$ inverts each element in $H_{1}$. But then $G$ is quasidihedral and so in particular all elements in $G-H_{1}$ must be involutions, a contradiction. We have proved that $\mathrm{Z}(G)=H_{1} \cap H_{2}$ is a maximal subgroup in $H_{1}$ and so $H_{2}$ is also a maximal subgroup in $G$.

If each minimal nonabelian subgroup in $G$ is isomorphic to $\mathrm{D}_{8}$, then by Theorem 10.33 in [1] our group $G$ is quasidihedral and so $G$ has only one maximal abelian subgroup of exponent $>2$, a contradiction. Hence $G$ possesses a minimal nonabelian subgroup

$$
M \cong \mathrm{M}_{2}(2,1,1)=\left\langle a, b \mid a^{4}=b^{2}=1,[a, b]=c, c^{2}=[c, a]=[c, b]=1\right\rangle .
$$

Then $M$ covers $G / H_{1}$ and $H_{1} / \mathrm{Z}(G)$ and $M \cap H_{1}$ is abelian of type $(4,2)$, where we have $M \cap \mathrm{Z}(G) \cong \mathrm{E}_{4}$. Indeed, if $M$ does not cover $G / H_{1}$ or $H_{1} / \mathrm{Z}(G)$, then $M$ would be abelian, a contradiction. Let $V$ be a complement of $M \cap \mathrm{Z}(G)$ in $\mathrm{Z}(G)$. Then $G=M \times V$ and our theorem is proved.

TheOREM 2. Let $G$ be a nonabelian p-group of exponent $>p$, where $p>2$. Suppose that $G$ has exactly $p$ maximal abelian subgroups $H_{1}, H_{2}, \ldots, H_{p}$ of exponent $>p$. Then $\exp (G)=p^{2}, \mathrm{Z}(G)$ is elementary abelian, each $H_{i}$ normalizes each $H_{j}(i, j=1,2, \ldots, p), H=H_{1} H_{2} \cdots H_{p}=\mathrm{H}_{p}(G)$ (Hughes subgroup) and $\mho_{1}(G) \leq \mathrm{Z}(H)=H_{1} \cap H_{2} \cdots \cap H_{p}$. 
Proof. Let $G$ be a $p$-group, $p>2$, satisfying the assumptions of Theorem 2. It is easy to see that $G$ possesses at least one minimal nonabelian subgroup $M$ which is isomorphic to $\mathrm{M}_{p^{3}}$ or $\mathrm{M}_{p}(2,1,1)$. Suppose that this is false. Then all minimal nonabelian subgroups of $G$ are isomorphic to $\mathrm{S}\left(p^{3}\right)$ and so by Proposition 7 in [3] $G$ has an abelian subgroup $A$ of exponent $>p$ and index $p$ such that $A=\mathrm{H}_{p}(G)$. But then $G$ has only one maximal abelian subgroup of exponent $>p$, a contradiction. Hence there is such $M$ as above. Any two maximal subgroups of $M$ lie in two distinct maximal abelian subgroups in $G$. In this way we get $p$ pairwise distinct maximal abelian subgroups in $G$ of exponent $>p$ and one maximal abelian subgroup which is elementary abelian. In particular, $\mathrm{Z}(G)$ is elementary abelian.

We want to show that $\exp (G)=p^{2}$. Let $H_{1}, H_{2}, \ldots, H_{p}$ be the set of all $p$ maximal abelian subgroups in $G$ which are of exponent $>p$. Set $\exp (G)=p^{e}$, where $e \geq 2$ and let $g$ be an element of order $p^{e}$ so that $g \in H=H_{1} H_{2} \cdots H_{p}$, where we know that each $H_{i}$ normalizes each $H_{j}$ (see the paragraph preceding Theorem 1). If $g$ is not contained in all $H_{i}(i=1,2, \ldots, p)$, say $g \notin H_{1}$, then by Lemma 57.1 in [2], there is $h_{1} \in H_{1}$ such that $\left\langle g, h_{1}\right\rangle$ is minimal nonabelian. Since all minimal nonabelian subgroups of $G$ are of exponent $\leq p^{2}$, we get $e=2$. So suppose that $g \in H_{i}$ for all $i=1,2, \ldots, p$. In particular, $g \in H_{1} \cap H_{2}$. Since $\left\langle H_{2}-H_{1}\right\rangle=H_{2}$, there is $h \in H_{2}-H_{1}$ such that $\mathrm{o}(h)=p^{e}$. By Lemma 57.1 in [2], there is $k \in H_{1}$ such that $\langle h, k\rangle$ is minimal nonabelian. This implies again $e=2$. We have proved that $\exp (G)=p^{2}$. If $H<G$, then all elements in $G-H$ are of order $p$ and so $H=\mathrm{H}_{p}(G)$. Now, $\mathrm{Z}(H)$ centralizes all $H_{i}$ and so $\mathrm{Z}(H) \leq H_{1} \cap H_{2} \cdots \cap H_{p}$. But $H_{1} \cap H_{2} \cdots \cap H_{p} \leq \mathrm{Z}(H)$ and so we get $\mathrm{Z}(H)=H_{1} \cap H_{2} \cdots \cap H_{p}$.

Let $g$ be any element of order $p^{2}$ in $G$. Then $g \in H=H_{1} H_{2} \cdots H_{p}$, where $H_{i} \unlhd H$ for all $i=1,2, \ldots, p$. We have either $g \in H_{i}$ (and then also $g^{p} \in H_{i}$ ) or (by Lemma 57.1 in [2]) there is $h_{i} \in H_{i}$ such that $M=\left\langle g, h_{i}\right\rangle$ is minimal nonabelian, where $M \cong \mathrm{M}_{p^{3}}$ or $M \cong \mathrm{M}_{p}(2,1,1)$. Then we know that $M$ contains exactly one maximal subgroup $X$ of exponent $p^{2}$ such that $X \leq H_{i}$. This implies that $g^{p} \in X \leq H_{i}$. Hence in any case we get $g^{p} \in H_{i}$ for all $i=1,2, \ldots, p$. Hence $g^{p} \in H_{1} \cap H_{2} \cdots \cap H_{p}=\mathrm{Z}(H)$ and so $\mho_{1}(G) \leq \mathrm{Z}(H)$. Our theorem is proved.

THEOREM 3. Let $G$ be a nonabelian 2-group with exactly 3 maximal abelian subgroups $H_{1}, H_{2}, H_{3}$ of exponent $>2$. Then $G=H_{1} H_{2} H_{3}$ and $\mathrm{Z}(G)=H_{1} \cap H_{2} \cap H_{3}$.

(a) If $H_{1}$ is conjugate in $G$ to (say) $H_{2}$, then $\exp \left(H_{1}\right)=4, H_{3}$ is of index 2 in $G$ with $\exp \left(H_{3}\right) \leq 8, \mathrm{Z}(G)$ is elementary abelian and $G$ has a maximal subgroup which is quasidihedral of exponent 4.

(b) If all $H_{i}$ are normal in $G, i=1,2,3$, then $G$ is of class $2, \mho_{1}(G) \leq$ $\mathrm{Z}(G)$ and so $G^{\prime}$ is elementary abelian. 
Proof. Let $G$ be a nonabelian 2-group with exactly 3 maximal abelian subgroups $H_{1}, H_{2}, H_{3}$ of exponent $>2$. Set $H=\left\langle H_{1}, H_{2}, H_{3}\right\rangle$ so that $H \unlhd G$. If $H<G$, then all elements in $G-H$ are involutions. But then (by a result of Burnside) $H$ is abelian, a contradiction. Hence we have $G=\left\langle H_{1}, H_{2}, H_{3}\right\rangle$ and then obviously $\mathrm{Z}(G)=H_{1} \cap H_{2} \cap H_{3}$.

(i) First we consider the case where some $H_{i}$ are not normal in $G$.

Then we may assume that $H_{1}$ and $H_{2}$ are conjugate in $G$ and then $H_{3} \unlhd G$. We set $K=\mathrm{N}_{G}\left(H_{1}\right)$ so that $|G: K|=2, H_{1}<K$ and $K=\mathrm{N}_{G}\left(H_{2}\right)$. For any $g \in G-K, H_{2}=H_{1}^{g}$ and $H_{1} H_{2}=H_{1}^{G}$. Then $H_{3}$ covers $G /\left(H_{1} H_{2}\right)$ so that $G=\left(H_{1} H_{2}\right) H_{3}$. All elements in $G-\left(K \cup H_{3}\right)$ are involutions and so for each involution $i \in G-\left(K \cup H_{3}\right)$, a maximal abelian subgroup in $G$ containing $i$ is elementary abelian. In particular, $\mathrm{Z}(G)$ is elementary abelian.

Set $G_{1}=H_{1} H_{3}$ and let $g \in H_{3}-K$ so that $H_{2}=H_{1}^{g} \leq G_{1}$. It follows $G_{1}=G$ and set $H_{3}^{*}=H_{3} \cap K$ so that $H_{3}^{*}$ normalizes $H_{1}$. We have $H_{1} \cap H_{3}=$ $\mathrm{Z}(G)$ is elementary abelian and also $H_{2} \cap H_{3}=\mathrm{Z}(G)$. Then $K=H_{1} H_{3}^{*}$ and

$$
K^{\prime} \leq H_{1} \cap H_{3}^{*}=\mathrm{Z}(G) \leq \mathrm{Z}(K)
$$

so that $K$ is of class 2 and $K^{\prime}$ is elementary abelian. For any $k_{1}, k_{2} \in K$ follows $\left[k_{1}^{2}, k_{2}\right]=\left[k_{1}, k_{2}\right]^{2}=1$ and so $\mho_{1}(K) \leq \mathrm{Z}(K)$. We have $\mathrm{Z}(K)<H_{1}$ and if $\mathrm{Z}(K)>\mathrm{Z}(G)$, then $\mathrm{Z}(K) H_{3}^{*}$ is contained in a maximal abelian subgroup in $G$ distinct from $H_{1}, H_{2}$ and $H_{3}$ and so $\mathrm{Z}(K) H_{3}^{*}$ must be elementary abelian. We have proved that in any case $\mathrm{Z}(K)$ is elementary abelian and so $\exp (K)=4$ and $4 \leq \exp \left(H_{3}\right) \leq 8$.

Assume, by way of contradiction, that $\mathrm{Z}(K)>\mathrm{Z}(G)$. Since $\mathrm{Z}(K)<H_{1}$, it follows that $L=\mathrm{Z}(K) H_{3}$ is a proper subgroup of $G$. We know that all elements in $G-(K \cup L)$ are involutions. Let $i \in G-(K \cup L)$ and $x \in K \cap L$. Then $i x \in G-(K \cup L)$ and so

$$
1=(i x)^{2}=i x i x \text { implying } x^{i}=x^{-1} .
$$

Since $i$ inverts each element in $K \cap L$, it follows that $i$ centralizes $\mathrm{Z}(K)$ (noting that $\mathrm{Z}(K)$ is elementary abelian). But then $\mathrm{Z}(K) \leq \mathrm{Z}(G)$, a contradiction. We have proved that $\mathrm{Z}(K)=\mathrm{Z}(G)$ and so in particular, $\mho_{1}\left(H_{1}\right) \leq \mathrm{Z}(G)$.

Suppose, by way of contradiction, that $H_{3}$ is not a maximal subgroup in $G$. Let $v$ be an element of order 4 in $H_{1}$ so that $v^{2} \in \mathrm{Z}(K)=\mathrm{Z}(G)$ and we set $R=H_{3}\langle v\rangle$. Since $\left|R: H_{3}\right|=2$, it follows that $R$ is a proper subgroup of $G$ and all elements in $G-(K \cup R)$ are involutions. If $i \in G-(K \cup R)$ and $y \in K \cap R$, then $i y \in G-(K \cup R)$ so that $i y$ is an involution implying $y^{i}=y^{-1}$. Thus $i$ inverts each element in $K \cap R=\langle v\rangle H_{3}^{*}$ implying that $K \cap R$ is abelian. Let $X$ be a maximal abelian subgroup of $G$ containing $K \cap R$. Since $X$ is obviously distinct from each $H_{i}, i=1,2,3$, and $\exp (X)>2$, we have a contradiction. We have proved that $H_{3}$ is a maximal subgroup in $G$.

All elements in $G-\left(K \cup H_{3}\right)$ are involutions, where $K$ and $H_{3}$ are two distinct maximal subgroups in $G$. Then each involution $i \in G-\left(K \cup H_{3}\right)$ 
inverts each element in $K \cap H_{3}=H_{3}^{*}$. In particular, $i$ centralizes $\Omega_{1}\left(H_{3}^{*}\right)$ and so $\Omega_{1}\left(H_{3}^{*}\right)=H_{1} \cap H_{3}=\mathrm{Z}(G)$. Since $H_{1} \cap H_{3}<H_{3}^{*}$, it follows that $\exp \left(H_{3}^{*}\right)=4$. Then $H_{3}^{*}\langle i\rangle$ is quasidihedral of exponent 4 and $H_{3}^{*}\langle i\rangle$ is a maximal subgroup in $G$. Finally, $H_{1} \cap H_{3}^{*}=\mathrm{Z}(G)$ is a maximal subgroup of $H_{1}$ and so $\exp \left(H_{1}\right)=4$ and $G=H_{1} H_{3}=H_{1} H_{2} H_{3}$. We have proved all properties of $G$ stated in part (a) of our theorem.

(ii) Now assume that all $H_{i}$ are normal in $G, i=1,2,3$.

Then we have again $G=H_{1} H_{2} H_{3}$.

(ii1) First suppose that $H_{1}, H_{2}$ and $H_{3}$ do not cover $G$.

Then $G-\left(H_{1} \cup H_{2} \cup H_{3}\right)$ is not empty so that all elements in $G-\left(H_{1} \cup\right.$ $\left.H_{2} \cup H_{3}\right)$ are involutions. Let $i \in G-\left(H_{1} \cup H_{2} \cup H_{3}\right)$ and let $A$ be a maximal abelian subgroup in $G$ containing $i$ so that $A$ is distinct from $H_{1}, H_{2}$ and $H_{3}$ implying that $A$ must be elementary abelian. Since $\mathrm{Z}(G)<A$, it follows that $\mathrm{Z}(G)$ is elementary abelian.

It is easy to see that $\exp (G)=4$. Suppose that $g \in G$ with $\mathrm{o}(g) \geq 8$. For any $i \in\{1,2,3\}$, we have either $g \in H_{i}$ (and then also $g^{2} \in H_{i}$ ) or $g \in G-H_{i}$. In the second case Lemma 57.1 in [2] implies that there is $h \in H_{i}$ such that $M=\langle g, h\rangle$ is minimal nonabelian. Since $\exp (M) \geq 8$, each of the three maximal subgroups $M_{i}(i=1,2,3)$ of $M$ are of exponent $>2$ and they lie in three pairwise distinct maximal abelian subgroups $H_{1}, H_{2}, H_{3}$ of exponent $>2$ in $G$. Hence for an $j \in\{1,2,3\}$, we have $M_{j} \leq H_{i}$ and then $g^{2} \in M_{j} \leq H_{i}$. We have proved that in any case $g^{2} \in H_{i}$ for each $i \in\{1,2,3\}$ and so $g^{2} \in H_{1} \cap H_{2} \cap H_{3}=\mathrm{Z}(G)$. But $\mathrm{Z}(G)$ is elementary abelian and so $\mathrm{o}\left(g^{2}\right) \leq 2$, a contradiction. We have proved that $\exp (G)=4$.

Suppose that there is $h \in G$ of order 4 such that $h^{2} \notin \mathrm{Z}(G)$. Since all elements of order 4 in $G$ are contained in $H_{1} \cup H_{2} \cup H_{3}$, we may assume that $h \in H_{1}$. Then interchanging $H_{2}$ and $H_{3}$ (if necessary), we may assume that $h^{2} \notin H_{2}$. Set $K_{0}=H_{1} H_{2}$ so that $\mathrm{Z}\left(K_{0}\right)=H_{1} \cap H_{2}$ and $h^{2} \notin \mathrm{Z}\left(K_{0}\right)$. We have $K_{0}^{\prime} \leq H_{1} \cap H_{2}=\mathrm{Z}\left(K_{0}\right)$ and so $K_{0}$ is of class 2. Suppose, by way of contradiction, that $\exp \left(\mathrm{Z}\left(K_{0}\right)\right)=4$. Let $k \in K_{0}-\left(H_{1} \cup H_{2}\right)$ and let $B$ be a maximal abelian subgroup of $G$ containing $\mathrm{Z}\left(K_{0}\right)\langle k\rangle$ so that we must have $B=H_{3}$. But then $H_{3} \geq \mathrm{Z}\left(K_{0}\right)$ and so $\mathrm{Z}\left(K_{0}\right)=H_{1} \cap H_{2} \cap H_{3}=\mathrm{Z}(G)$, a contradiction. Hence $\mathrm{Z}\left(K_{0}\right)$ is elementary abelian. But then for all $x \in K_{0}$, $\left[h^{2}, x\right]=[h, x]^{2}=1$ and so $h^{2} \in \mathrm{Z}\left(K_{0}\right)$, a final contradiction. We have proved that $\mho_{1}(G) \leq \mathrm{Z}(G)$ implying that $G^{\prime}$ is elementary abelian and so we have obtained some 2-groups from part (b) of our theorem.

(ii2) Now assume that $G=H_{1} \cup H_{2} \cup H_{3}$, i.e., $H_{1}, H_{2}, H_{3}$ cover $G$.

Let $i \neq j$ with $i, j \in\{1,2,3\}=\{i, j, k\}$. If $H_{i} H_{j}<G$, then $H_{k} \geq$ $G-\left(H_{i} H_{j}\right)$ and since $\left\langle G-\left(H_{i} H_{j}\right)\right\rangle=G, G$ would be abelian, a contradiction. Thus

$$
H_{i} H_{j}=G, H_{i} \cap H_{j}=\mathrm{Z}(G), H_{k} \geq G-\left(H_{i} \cup H_{j}\right) \text { and } H_{k} \geq \mathrm{Z}(G) .
$$


Because $i \neq j$ are arbitrary elements in $\{1,2,3\}$, we also get

$$
H_{i} \cap H_{k}=H_{j} \cap H_{k}=\mathrm{Z}(G) \text { and so } H_{k}=\left(G-\left(H_{i} \cup H_{j}\right)\right) \cup \mathrm{Z}(G) .
$$

Also, $G^{\prime} \leq H_{i} \cap H_{j}=\mathrm{Z}(G)$ and so $G$ is of class 2 .

If $\mathrm{Z}(G)$ is elementary abelian, then for any $x, y \in G,\left[x^{2}, y\right]=[x, y]^{2}=1$ and so $\mho_{1}(G) \leq \mathrm{Z}(G)$. So assume that $\exp (\mathrm{Z}(G))>2$. In this case each maximal abelian subgroup of $G$ contains $\mathrm{Z}(G)$ and so must be equal to one of $H_{1}, H_{2}, H_{3}$. Let $g \in G$. Then either $g \in H_{i}$ (and then also $g^{2} \in H_{i}$ ) or $g \in G-H_{i}$. In the second case, by Lemma 57.1 in [2], there is $h \in H_{i}$ such that $M=\langle g, h\rangle$ is minimal nonabelian. Then three maximal subgroups $S_{1}, S_{2}, S_{3}$ of $M$ lie in three pairwise distinct maximal abelian subgroups in $G$ which are equal to $H_{1}, H_{2}$ or $H_{3}$. Hence we may assume $S_{1} \leq H_{i}$ and so $g^{2} \in H_{i}$. Thus in any case, $g^{2} \in H_{1} \cap H_{2} \cap H_{3}=\mathrm{Z}(G)$ and so we get again $\mho_{1}(G) \leq \mathrm{Z}(G)$. For any $x, y \in G,[x, y]^{2}=\left[x^{2}, y\right]=1$ and so $G^{\prime}$ is elementary abelian. We have obtained the groups from part (b) of our theorem and we are done.

\section{REFERENCES}

[1] Y. Berkovich, Groups of prime power order, Vol. 1, Walter de Gruyter, Berlin-New York, 2008.

[2] Y. Berkovich and Z. Janko, Groups of prime power order, Vol. 2, Walter de Gruyter, Berlin-New York, 2008.

[3] Z. Janko, Finite p-groups with some isolated subgroups, J. Algebra 465 (2016), 41-61.

Z. Janko

Mathematical Institute

University of Heidelberg

69120 Heidelberg

Germany

E-mail: janko@mathi.uni-heidelberg.de

Received: 25.7.2016. 